Nouvelles perspectives en sciences sociales

Revue internationale de systémique complexe et d'études relationnelles

\title{
L’Économie turquoise, entre territoire de projet politique et représentations territoriales différenciées : le devoir d'articulation
}

\section{Marion Bourhis}

Volume 10, numéro 1, novembre 2014

Sur le thème du territoire

URI : https://id.erudit.org/iderudit/1028439ar

DOI : https://doi.org/10.7202/1028439ar

Aller au sommaire du numéro

\section{Éditeur(s)}

Prise de parole

ISSN

1712-8307 (imprimé)

1918-7475 (numérique)

Découvrir la revue

Citer cet article

Bourhis, M. (2014). L'Économie turquoise, entre territoire de projet politique et représentations territoriales différenciées : le devoir d'articulation. Nouvelles perspectives en sciences sociales, 10(1), 163-191.

https://doi.org/10.7202/1028439ar

\section{Résumé de l'article}

Bien souvent convoqué comme support de mobilisation de l'action collective, le territoire n'en est pas moins polysémique dans sa définition. Le Conseil Général des Côtes-d'Armor en tant que collectivité territoriale et puissance publique s'appuie sur le territoire administratif relevant de sa prérogative pour mettre en oeuvre sa démarche Économie turquoise. Cette dernière a pour objectif de réaffirmer et de renforcer l'ambition maritime des Côtes-d'Armor tout en promouvant une diversification économique se basant sur une gestion intégrée de l'espace et des ressources maritimes et littorales. Or, bien que le Conseil général soit perçu comme légitime, en tant que puissance publique, pour intervenir; le choix du territoire d'intervention a bien souvent été posé par les acteurs, qu'ils participent ou non à la démarche. En nous basant sur nos travaux de recherche, nous analyserons les raisons de ce décalage et démontrerons comment ce dernier peut être dépassé grâce à une action publique pensée dans une perspective interterritoriale. 


\title{
L'Économie turquoise, entre territoire de projet politique et représentations territoriales différenciées : le devoir d'articulation
}

\author{
MARION BOURHIS \\ Institut de Géoarchitecture, \\ Université de Bretagne-Sud, Lorient
}

e Conseil général des Côtes-d'Armor en tant que collectivité sa prérogative pour construire sa démarche Économie turquoise. Celle-ci a pour objectif de réaffirmer et de renforcer l'ambition maritime des Côtes-d'Armor tout en promouvant une diversification économique se basant sur une gestion intégrée de l'espace et des ressources maritimes et littorales. Au travers de l'Économie turquoise on peut noter l'intérêt croissant porté par le Département à son littoral et à son espace maritime comme potentiellement porteurs de développement de nouvelles activités. Loin de lui être spécifique, cet intérêt s'inscrit dans une évolution plus globale, perceptible notamment jusqu'à l'échelle européenne (Politique Maritime Intégrée, stratégie "Croissance Bleue», etc.). En cela, elle constitue une politique volontariste recherchant à instituer à l'échelle départementale un nouvel espace ${ }^{1}$ de discussion et

Le Département possède peu de compétences identifiées comme maritimes : gestion de ports de commerce, de ports de pêche (dont certains possèdent aujourd'hui une forte dominante plaisancière), gestion des transports portuaires (desserte des îles, police portuaire, etc.). Néanmoins, il possède des 
d'action collective sur la mer et le littoral en intégrant des préoccupations globales au niveau local. Le département devient alors le territoire sur lequel le projet peut s'ancrer, réunissant des acteurs hétérogènes possédant de multiples appartenances institutionnelles et développant des intérêts diversifiés. Pourtant, le département, comme échelle d'action sur l'espace maritime et littoral pour mettre en œuvre l'Économie turquoise, ne rencontre pas automatiquement l'adhésion des acteurs locaux. En effet, alors même qu'au travers de l'Économie turquoise, le département est présenté comme territoire pertinent de projet pour l'action du point de vue autant de sa délimitation que de ses compétences, et l'action comme s'articulant avec les autres démarches (Politique maritime intégrée, Charte des espaces Côtiers Bretons, etc.); les acteurs, qu'ils aient été conviés ou non à participer à la démarche, indiquent que les espaces maritimes et littoraux, relèvent de compétences multiples attachées à différents niveaux de collectivités. D'où vient ce décalage de représentations? Comment s'explique-t-il?

Nous émettons deux hypothèses. La première porte sur des définitions différenciées du territoire, selon les acteurs, en fonction des pratiques et des échelles de gestion. La seconde est qu'il n'existe pas d'échelles ou de territoires pertinents a priori mais seulement des politiques en capacité de combiner les échelles et de mobiliser les acteurs et les territoires ensemble dans une interterritorialité de l'action. Celle-ci représente le passage de territoires organisant et exerçant le pouvoir sur l'étendue qu'ils délimitent chacun en et pour eux-mêmes à l'organisation et l'exercice d'un pouvoir territorial partagé entre ces derniers ${ }^{2}$. Afin d'étayer et d'illustrer notre propos, nous basons notre analyse sur une méthode alliant la réalisation d'entretiens semi-directifs ${ }^{3}$ à

compétences impactant l'espace maritime et le littoral, comme, par exemple, la gestion de l'eau. À ces dernières s'ajoutent des politiques volontaristes.

2 Martin Vanier, Le pouvoir des territoires : essai sur l'interterritorialité, préface de Philippe Estèbe, Paris, Economica Anthropos, 2008.

3 Les enquêtés appartiennent à quatre catégories d'acteurs : ceux ayant été invités et présents aux réunions de l'Économie turquoise, ceux ayant été invités et non présents, ceux ayant été envisagés pour être invités et qui ne l'ont jamais été et, enfin, ceux non invités mais présents au cours du proces- 
une analyse documentaire (revue de presse, documents évoquant l'Économie turquoise, documents produits au sein de la démarche) et à l'observation (parfois participante) de la démarche en construction. De plus, notre recherche s'inscrivant dans le cadre d'une CIFRE (Convention Industrielle de Formation par la REcherche) a permis de négocier une présence au plus près du terrain d'étude.

Notre propos s'attachera premièrement à présenter la démarche Économie turquoise, en tant que projet de territoire à l'échelle départementale (1). Puis, nous analyserons les représentations territoriales sur lesquelles se base la mise en ouvre de cette politique par le Conseil général (2) tout en démontrant qu'elle met en jeu des territoires de pratiques et d'actions différenciés selon les acteurs (3). Enfin, nous démontrerons en quoi l'interterritorialité peut être présentée comme une réponse permettant de dépasser les écueils rencontrés (4).

\section{L'Économie turquoise, un projet de territoire à l'échelle départementale}

Territoire au passé maritime, mais majoritairement perçu par ses habitants comme agricole ${ }^{4}$ (au-delà d'espaces récréatifs et de tourisme), le Département des Côtes-d'Armor tente de renforcer et de réaffirmer son ambition maritime. Ainsi, le discours politique du Conseil général valorise ses ports de pêche, de commerce et de plaisance, son potentiel en termes d'énergies marines renouvelables ou encore de gestion de son gisement de coquilles Saint-Jacques. L'affirmation de cette ambition se trouve aujourd'hui incarnée par l'Économie turquoise, politique volontariste du Conseil général.

sus. Les enquêtés interrogés sont des élus, des techniciens des services, des membres des secteurs économiques ou associatifs.

4 Ce constat est issu de la démarche Côtes d'Armor 2mille20. Débutée en 2006, elle avait pour objectif de construire une vision partagée de l'avenir du département et d'organiser le débat entre élus, membres de la société civile et citoyens. Dans ce cadre, de nombreux questionnaires ont été distribués et des entretiens ont été menés. Certains permettaient d'analyser la représentation de l'espace maritime et littoral du département. 


\subsection{Un contexte européen et national}

Autrefois considérés comme répulsifs, depuis devenus attractifs, les espaces littoraux et maritimes, dans le rapport que la société entretient avec eux, ont connu nombre d'évolutions. Considérés tantôt comme espaces mythiques ${ }^{5}$, puis comme espaces récréatifs ${ }^{6}$, les représentations qui y sont associées tendent à se modifier ${ }^{7}$. Depuis les années 1970, période à laquelle une première prise de conscience généralisée des problèmes pouvant se poser sur le littoral se fait ressentir, la représentation de ces espaces a évolué d'une vision "protectionniste» à une vision "d'aménageur». Ce n'est qu’à partir des années 1980 , et plus encore après 1990, que la nécessité d'une gestion intégrée des zones côtières ${ }^{8}$, qui prenne en compte l'ensemble des enjeux maritimes et terrestres des littoraux en associant l'ensemble des acteurs, se fait jour et s'impose progressivement ${ }^{9}$. Depuis maintenant moins d'une décennie, semble se profiler, à l'échelle européenne, un nouveau changement. En effet, en parallèle de l'adoption de la Directive «Planification spatiale maritime et gestion intégrée de la zone côtière» (DCSMM) constituant le volet environnemental de la Politique Maritime Intégrée (PMI), la Commission Européenne réalise en 2012 une étude intitulée "Croissance Bleue : des possibilités de croissance durable dans les secteurs marin et maritime ${ }^{10}$ ». Conçue comme la déclinaison économique de la PMI et dimension maritime de la Stratégie Europe 2020,

5 Michel Roux, L'imaginaire marin des Français : mythe et géographie de la mer, Paris, L'Harmattan, 2000.

6 Jean-Marie Bécet, L'aménagement du littoral, Paris, Presses universitaires de France, coll. "Que sais-je?», 1987.

7 Françoise Péron et Jean Rieucau, La maritimité aujourd'hui, Paris, L'Harmattan, 1996.

8 Pour définition, se reporter à Olivier Lozachmeur, "Le concept de gestion intégrée des zones côtières : le point de vue d'un juriste» (Océanis, vol. 30, $\mathrm{n}^{\circ}$ 1, 2004, p. 5170) ainsi qu'à Conseil de l'Europe, Modèle de loi sur la gestion durable des zones côtières et Code de conduite européen des zones côtières (1999).

$9 \quad$ Alain Merckelbagh, Et si le littoral allait jusqu’à la mer!, Versailles, Editions Quae, 2009.

10 Commission européenne, Blue Growth : Opportunities for Marine and Maritime Sustainable Growth, Bruxelles, Direction générale des affaires maritimes et de la pêche, 2012. 
celle-ci reconnait le couple mer/littoral comme faisant partie des moteurs de l'économie européenne et comme source d'innovation et de croissance. L'adoption de la «DCSMM» et de la stratégie «Croissance Bleue» représente donc une nouvelle évolution. Y est présente la volonté de concilier la protection des espaces maritimes et littoraux et le développement économique de ces espaces, conception qui se trouve être au cœur de l'élaboration de la politique Économie turquoise du Conseil général.

\subsection{La formulation progressive d'une ambition}

L'Économie turquoise, en écho aux doctrines énoncées à des échelles plus globales, a fait l'objet d'une évolution de sa formulation depuis la fin des années 1990 (voir la figure 1).

Figure 1

Formulations successives d'une ambition maritime et littorale par le Conseil général des Côtes-d'Armor

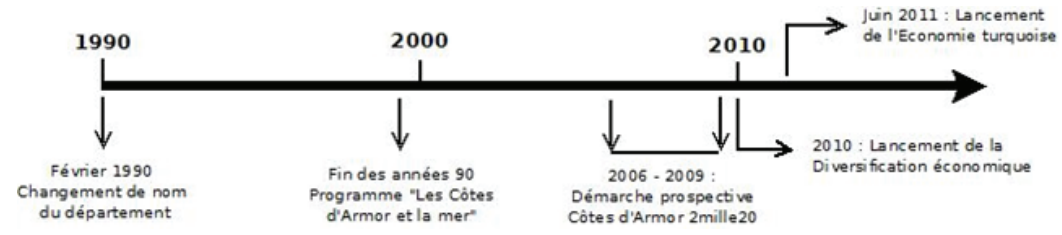

Ainsi, le programme "Les Côtes-d'Armor et la mer», réalisé en 2000 par le Conseil général des Côtes-d'Armor, a posé les grands principes de l'engagement et de l'action de ce dernier. Très orienté sur les enjeux patrimoniaux et portuaires, ce premier programme a permis la réalisation d'actions telles qu'un inventaire du patrimoine maritime et littoral des Côtes-d'Armor ou encore la réalisation d'infrastructures portuaires. En 2006, la démarche prospective "Côtes d'Armor 2 mille 20 » a prolongé le travail entamé au travers de son «initiative thématique Mer et Littoral $^{11}$ » et du document « Pour une Stratégie Départementale

11 Conseil général des Côtes-d'Armor, Initiative thématique "Mer et Littoral», Saint-Brieuc, Côtes d'Armor 2mille20, 2006. 
de Recherche et d'Innovation en Côtes d'Armor ${ }^{12}{ }$. Enfin, depuis 2011, le Conseil général s'est engagé dans une politique de «Diversification économique». Cette dernière a pour objectif d'encourager les collaborations entre les acteurs économiques du département représentés notamment par la Chambre de commerce et d'industrie des Côtes-d'Armor (CCI22), l'Agence de développement économique "Côtes-d'Armor Développement " (CAD22), la Chambre d'agriculture, des technopôles (Anticipa, Zoopôle), ou des associations (ITS Bretagne ${ }^{13}$, par exemple). L'Économie turquoise, qui en est un des axes de travail, s'inscrit dans les réflexions précédentes tout en les prolongeant. Définie comme «une stratégie opérationnelle dans le domaine de la mer, de l'écologie et de l'économie marine ${ }^{14}$ ", l'Économie turquoise a vocation à expérimenter de nouvelles voies économiques maritimes et littorales innovantes qui soient porteuses de richesses et d'emplois pour le territoire costarmoricain. Pour cela, elle doit "promouvoir une diversification économique [...]. Une économie "Glaz" en somme. "Glaz" qui signifie à la fois vert et bleu en breton ${ }^{15}$ ". La démarche doit donc participer au renouvellement de l'ambition du Département en nourrissant «l'intention d'une transformation du réel, guidée par une représentation du sens de cette transformation ${ }^{16}$ ».

12 Au cours de la démarche «Côtes d'Armor 2 mille 20 », nombre de questions sont restées sans réponse dans le domaine de la recherche et de l'innovation, d'où une prolongation de la réflexion au travers du rapport "Contribution à une stratégie départementale de Recherche et d'Innovation en Côtesd'Armor" (2011).

13 ITS Bretagne est une association Loi 1901 dont l'objet est de promouvoir et d'assurer la coordination d'un pôle de compétences axé sur le développement des Systèmes de Transports Intelligents ou Intelligent Transport Systems (ITS).

14 Conseil général des Côtes-d'Armor, Faire de la mer et du littoral une force pour notre département, Budget primitif 2012, Saint-Brieuc, Séances des 27, 28 février, 1 et 2 mars 2012 .

15 Conseil général des Côtes-d'Armor, Économie turquoise. Rapport d'orientations, Saint-Brieuc, Direction de la mer et du littoral, $n^{\circ} 4.5$, séances des 22 et 23 septembre 2011, p. 4.

16 Jean-Pierre Boutinet, Anthropologie du projet, Paris, Presses universitaires de France, coll. «Quadrige», 2012, p. 146. 
1.3. Un territoire de projet ne faisant pas l'objet d'un large partage

Avec pour objectif de préciser l'ambition poursuivie au travers de l'Économie turquoise, la production d'un rapport d'orientations a été présentée en Assemblée générale départementale en septembre 2011. En donnant les grandes lignes de la démarche ainsi que les enjeux identifiés, ce rapport a construit le cadre d'inscription de la démarche dans sa construction et sa mise en œuvre ultérieure. Sur cette base, des groupes de travail élargis ont été organisés avec pour objectif d' "élaborer un diagnostic sur la base d'un partage de connaissances ${ }^{17}$ ", $d^{\prime}$ " "analyser de manière détaillée des enjeux ${ }^{18}$ ", et d' "alimenter un programme d'actions en cours d'élaboration" pour permettre une transcription "concrète» et "opérationnelle» de l'Économie turquoise ${ }^{19}$. Conçus à géométrie variable, ces groupes ont convoqué dans leur grande majorité des acteurs costarmoricains (collectivités territoriales, associations, entreprises, etc.), à quelques exceptions près : la Région Bretagne, Bretagne Prospective (une association de réflexion prospective sur l'avenir du territoire breton), le Pôle Mer Bretagne Atlantique (PMBA) et la démarche GIZC Rance-Côte d'Émeraude (à cheval sur les départements de l'Ille-et-Vilaine et des Côtes-d'Armor, mais néanmoins d'une superficie infra-départementale).

Par la suite, un comité de pilotage (COPIL) a été mis en place pour assurer le suivi global de la démarche, des actions et des axes d'expérimentations issus du programme d'actions (axes validés par la suite par l'exécutif du Conseil général) :

- les ports durables : engager une réflexion sur la définition même d'un port durable, intégrer les éléments de réflexion lors des renouvellements futurs de délégations de service public, etc.;

17 Conseil général des Côtes-d'Armor, Compte-rendu du groupe de travail élargi «Économie turquoise», Saint-Brieuc, 18 octobre 2011.

18 Conseil général des Côtes-d'Armor, Compte-rendu du groupe de travail élargi "Économie turquoise», Saint-Brieuc, 12 janvier 2012.

19 Conseil général des Côtes-d'Armor, Compte-rendu du groupe de travail élargi «Économie turquoise», Saint-Brieuc, 10 juillet 2012. 
- les énergies marines renouvelables : supporter les différentes initiatives pour en faire profiter le territoire;

- la diversification de l'aquaculture : maintenir les filières traditionnelles déjà existantes, tout en les diversifiant;

- mer et citoyenneté : changer le regard des costarmoricains sur la mer pour que cet espace ne soit plus seulement représenté comme un espace majoritairement de loisirs, mais aussi comme un espace de développement économique d'activités présentes et futures;

- l'organisation d'un Forum de la mer : instaurer un lieu de discussion et d'action collective réunissant l'ensemble des acteurs concernés.

Le COPIL est constitué à la fois d'acteurs départementaux et d'acteurs extra-départementaux. Parmi les premiers, on peut citer le Conseil général ainsi qu'un centre technique (le CEVA : Centre d'Étude et de Valorisation des Algues) implanté dans le TrégorGoëlo (Ouest du département), CAD22, les démarches «Gestion Intégrée de la Zone Côtière» (GIZC) Baie de Saint-Brieuc et Trégor-Goëlo, la Chambre de commerce et d'industrie des Côtes-d'Armor (CCI 22) et l'Agence de développement industriel du Trégor. Les seconds sont constitués par Bretagne Prospective, la démarche GIZC Rance-Côte d'Émeraude et la Région Bretagne. C'est donc une certaine pluralité d'acteurs présents et pressentis qui peut être observée au travers de l'Économie turquoise et qui a engendré une pluralité d'usages du territoire, de territorialisation et de représentations qu'il nous faut alors analyser.

\section{Un territoire d'action départemental pour une échelle d'intervention départementale}

L'Économie turquoise, dans sa conception, constitue une démarche initiée dans une logique "top-down" par le Conseil général. En recherche d'un ancrage local, les acteurs se sont vus mobilisés au travers de groupes de travail élargis et d'un COPIL. Pour autant, l'ensemble des éléments constitutifs de l'Économie 
turquoise n'a pas fait l'objet d'une construction commune telle qu'elle aurait pu être idéalement souhaitée. C'est, par exemple, la question du périmètre d'intervention qui, au final, est le département, donné comme un intangible de la démarche et comme étant un espace homogène pertinent de mise en œuvre auquel a été adjoint l'espace littoral et maritime.

2.1. Une conception du département comme espace homogène

Le département résulte d'un découpage spatial sur lequel une autorité publique est reconnue comme étant légitime. L'étendue du territoire définit alors le champ d'application du pouvoir. Dans le cadre de l'Économie turquoise, le Conseil général se base justement sur le territoire départemental comme périmètre de l'action, donc support de l'action collective allant de soi. Le territoire d'action qu'est le département est alors compris dans le sens d'une définition se reposant tout à la fois sur les notions d'administration et de gestion d'un espace très clairement délimité $^{20}$. C'est donc une entrée enfermant les territoires dans des limites administratives rigides ${ }^{21}$, objet du lieu d'exercice d'une domination, d'une légitimité ou d'une souverainetée ${ }^{22}$ qui est privilégiée. De plus, en s'appuyant sur le territoire départemental pour incarner son action publique, le Conseil général se place au-dessus des intérêts individuels et garant des principes d'égalité $^{23}$ et de non-concurrence. La mise en œuvre de l'Économie turquoise à cette échelle revient alors à garantir ces principes, mais aussi à considérer le département comme un espace homogène au sein duquel ne transparaît pas de disparités ou de spécificités.

$20 \quad$ Alexandre Moine, "Comprendre et observer les territoires : l'indispensable apport de la systémique», Habilitation à Diriger des Recherches, Université de Franche-Comté, 2014.

21 Ibid.

22 Françoise Gerbaux (dir.), Utopie pour le territoire : cohérence ou complexité, La Tour-d'Aigues, Éditions de l'Aube, 1999.

23 Rosaire Gob, «Le management du territoire par le projet : des limites de l'instrumentation gestionnaire. Le cas de la Guadeloupe», Thèse, Conservatoire National des Arts et Métiers, 2012. 
Ainsi, le territoire départemental, au sens juridico-administratif du terme, fait partie intégrante de l'identité du projet. Pour autant, l'Économie turquoise possède une particularité, celle d'être un projet portant sur des problématiques maritimes et littorales. En ce sens, l'acceptation première de ce qu'est le territoire départemental ne peut y répondre. Le département s'est alors vu adjoindre l'espace maritime et littoral dès la conception de l'Économie turquoise.

2.2. La construction d'un nouveau territoire administratif sur des bases départementales

Dès 2011, dans son rapport d'étape sur l'Économie turquoise, le Conseil général affirme la légitimité du Département à se doter d'une ambition maritime. Il va même au-delà en parlant de "territoire maritime des Côtes-d'Armor ${ }^{24}$ " lorsqu'il évoque le potentiel en matière de nouvelles voies de développement des activités existantes et de nouvelles économies marines. Le projet fait alors référence à des enjeux concernant à la fois l'espace maritime (Domaine Public Maritime (DPM)), le littoral, le rétro-littoral et l'arrière-pays. Or, la gestion du DPM relève de l'État, celle du littoral varie selon la définition donnée de ce dernier (voir la figure 2).

24 Conseil général des Côtes-d'Armor, Économie turquoise. Rapport d'orientations, op. cit., p. 3. 
Figure 2

Limites administratives et chevauchements des domaines de compétences sur la frange littorale (Source : Parc marin d'Iroise, 2006)

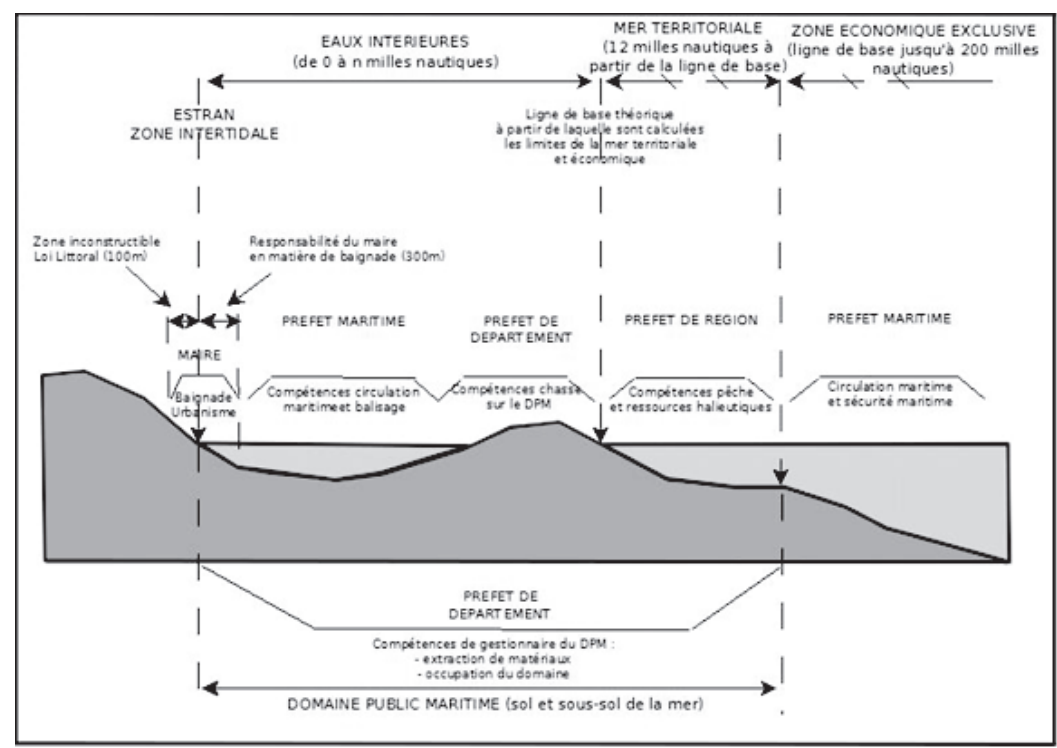

On peut alors comprendre la difficulté pour le Conseil général d'intervenir sur les enjeux définis au sein de l'Économie turquoise. Cette difficulté est due en partie à la question des limites spatiales du département qui interfèrent sur les limites des compétences. Alors que le département existe déjà en tant que territoire administratif, on observe la construction d'un nouveau territoire administratif sur des bases départementales par une annexion en cours de l'espace maritime ne relevant pourtant pas d'une gestion par la collectivité publique qu'est le Département. L'annexion de cet espace peut alors relever d'une stratégie, de la part du Conseil général, de justification de son intervention. Néanmoins, l'annexion reste floue, jamais illustrée ni cartographiée et sans aucune indication sur l'étendue des prérogatives. Ce territoire, «en train de se faire», ne bénéficie pas de véritable délimitation, 
ni d'organisation ou de dénomination ${ }^{25}$ mais devient néanmoins visible $^{26}$ par le biais de l'Économie turquoise ${ }^{27}$.

Cette annexion pose donc question. Remonte-t-elle à 2011 avec la dynamique enclenchée au sein de l'Économie turquoise ou a-t-elle, elle aussi, fait l'objet d'un processus sur le temps long comme la formulation d'une ambition maritime par le Département? Toujours est-il que le département des Côtesd'Armor n'est alors plus seulement terrestre mais aussi maritime, dans un souci de légitimation du territoire d'action publique départemental comme pertinent pour mettre en ouvre l'Économie turquoise. Pourtant, cette représentation n'est pas véritablement partagée.

\section{Les territoires des acteurs socio-économiques conviés ou non}

Nous avons pu analyser le territoire de référence de l'Économie turquoise sur lequel se base le Conseil général grâce à l'analyse des discours politiques et des productions écrites techniques au sein de la démarche. Pour comprendre les décalages observés, nous nous sommes appuyés sur des entretiens semi-directifs ayant porté, entre autres, sur les représentations du territoire et la légitimité du Conseil général à mener une politique telle que l'Économie turquoise. Nous avons interrogé des élus, des techniciens, des associations, des responsables d'entreprises, des représentants d'usagers, des représentants du secteur économique, etc. Quelle(s) représentation(s) ont-ils du territoire de mise en œuvre? En quoi ces représentions divergent-elles? Sur la base de quels critères?

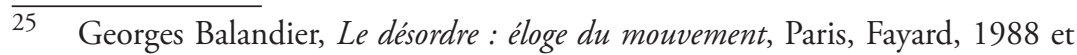
Anthropologie politique, Paris, Presses universitaires de France, coll. "Quadrige», 1995.

26 Romain Lajarge, "Pas de territorialisation sans action (et vice versa)?", dans Martin Vanier (dir.), Territoires, territorialité, territorialisation: controverses et perspectives actes des entretiens de la Cité des territoires, Grenoble, 7 et 8 juin 2007, "Territoires, territorialité, territorialisation... et après?", Rennes, Presses universitaires de Rennes, 2009, p. 193-204. Hervé Gumuchian et al., Les acteurs, ces oubliés du territoire, Paris, Economica Anthropos, 2003. 
3.1. Une représentation du territoire selon une vision actantielle et relationnelle

Alors que le département est présenté comme territoire pertinent de mise en œuvre de l'Économie turquoise, certains acteurs se réferent à l'existence de territoires d'usages, de territoires vécus, pour définir le leur. Engager une politique à l'échelle départementale est alors parfois jugé comme manquant de cohérence et déconnecté des réalités territoriales : "L'Économie turquoise c'est une initiative louable sur un territoire qui n'est pas forcément cohérent» (Chargé de mission, Association). En effet, dans les entretiens, le département a été décrit comme composé de trois sous-ensembles géographiques, ne respectant pas strictement les limites administratives départementales, difficiles à articuler et aux pratiques et aux enjeux différents : la Baie de Saint-Brieuc, l'Ouest du département (à partir de Paimpol, sans précisions quant à la limite donnée), la Baie de Saint-Malo à cheval entre les départements des Côtes-d'Armor et de l'Ille-et-Vilaine (pour la pêche, ce sous-ensemble va au-delà de l'Ille-et-Vilaine). Ces sous-ensembles ont été décrits par les enquêtés comme au plus proche des masses d'eau et des écosystèmes ainsi que des territoires d'usages. Les exemples de la plaisance $^{28}$ et de la pêche ont souvent été cités pour appuyer ces propos. Les territoires auxquels se réferent ici les acteurs interrogés ne sont donc pas seulement des entités géographiques mais le fruit de productions collectives. L'espace défini par l'Économie turquoise ne coïncide alors pas avec celui des pratiques de coopération et de sociabilité ${ }^{29}$ déjà existantes.

Les acteurs exprimant cette vision possèdent une échelle d'intervention plus locale que l'échelle départementale. C'est

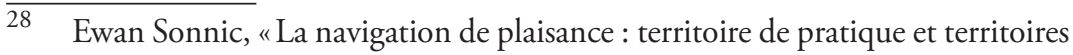
de gestion en Bretagne, entre dualité et nécessité de fusion pour une évolution progressiste de l'activité», Thèse, Université de Rennes 2, 2005. Les concepts de bassin de plaisance et bassin de navigation y étaient évoqués.

Valérie Angeon et Nathalie Bertrand, Les dispositifs contractuels de développement rural : quelles proximités mobilisées?, Colloque ASRDLF sur Les dynamiques territoriales : débats et enjeux entre les différentes approches disciplinaires, Grenoble-Chambery, 10-12 juillet, 2007. 
notamment le cas d'acteurs menant des initiatives locales ou y étant associés, comme les démarches de projets "GIZC». Il est intéressant de noter, concernant ces démarches, que leur territoire d'action est celui qui se rapproche le plus des sous-ensembles géographiques évoqués dans le cadre des entretiens comme le montre la figure 3 ci-dessous.

Figure 3

Sous-ensembles géographiques et démarches GIZC concernant le territoire

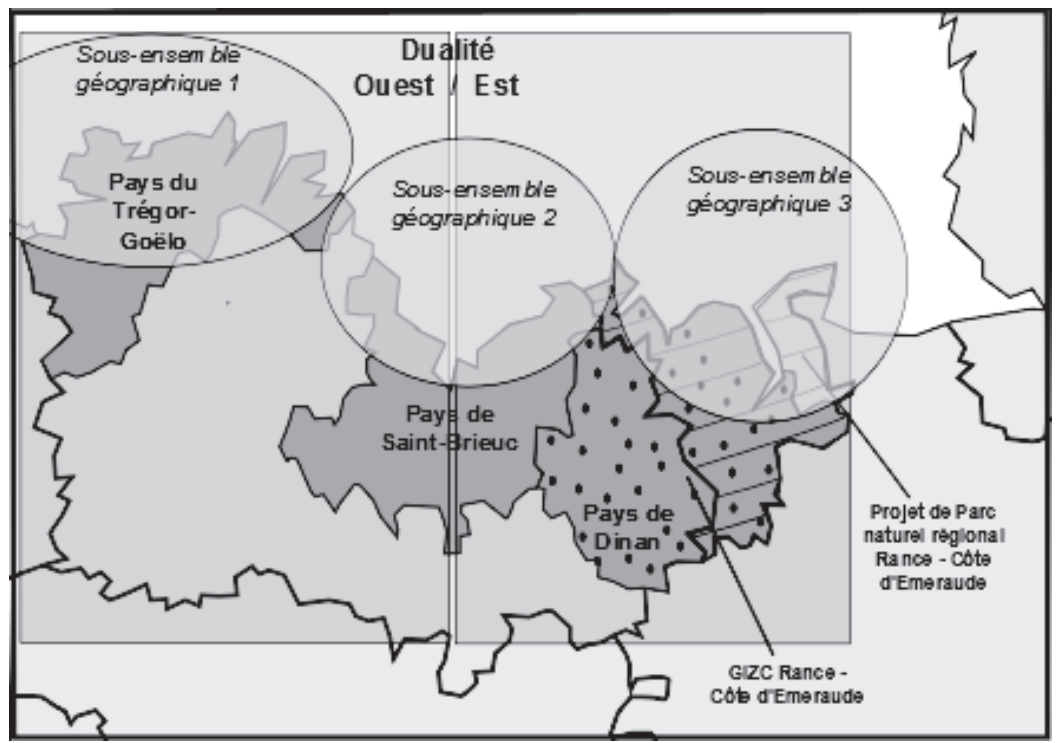

Deux autres exemples viennent renforcer l'existence de sousensembles. Dans le cadre des entretiens, nous avons été interpellés sur la nécessité d'interroger autant d'élus à l'Ouest et à l'Est du département. Une réelle dualité existe. La bataille pour l'obtention du port de maintenance du parc éolien offshore, qui doit être construit en Baie de Saint-Brieuc, en est un exemple frappant. Le choix du port devait être acté par le consortium portant le projet de parc. Le Conseil général devait identifier des ports pouvant répondre aux besoins et organiser le débat. Trois ports ont été identifiés : Saint-Quay-Portrieux (versant Ouest de la Baie 
de Saint-Brieuc), Erquy (versant Est de la Baie de Saint-Brieuc) et Saint-Cast-du-Guildo (Est du département, hors de la baie). Pendant plusieurs mois, les élus ont défendu «leurs» ports respectifs. Rapidement, Erquy s'est rallié à Saint-Cast-du-Guildo, l'enjeu étant que ce soit l'Est du département qui récupère cet équipement. Néanmoins, Saint-Quay-Portrieux fut retenu. Bien que le choix fut officiellement celui du consortium, des voix s'élevèrent officieusement pour dénoncer un choix réalisé dès le départ et privilégiant l'Ouest. Cet exemple révèle bien l'existence de deux systèmes spatiaux en termes de proximités géographiques et organisées, au sens d'André Torre et Jean-Eudes Beuret ${ }^{30}$. Un indice supplémentaire de cette partition réside dans les faits suivants. Dans le cadre de l'Économie turquoise, le Conseil général souhaite renforcer son partenariat avec le PMBA dans l'objectif de dynamiser le tissu d'entreprises costarmoricaines et l'inciter à innover dans les technologies afférentes à l'espace maritime et littoral. À cet effet, le PMBA s'appuie sur des relais territoriaux. Sur le territoire costarmoricain, le seul relais existant se charge de cette mission exclusivement sur l'Ouest du département. Â nos interrogations relatives à cette situation, tous, que ce soit la personne relais, les acteurs économiques, les techniciens ou les élus, ont relevé la pertinence de l'action sur l'Ouest de la personne relais, mais aussi son manque de connaissance du tissu existant sur la baie de Saint-Brieuc et à l'Est du département, tout comme son manque de légitimité et de réseau pour y intervenir.

Ainsi, c'est l'existence non pas d'une échelle d'action mais d'échelles d'actions englobées qui est révélée avec la nécessité de

$\overline{30}$ Selon André Torre et Jean-Eudes Beuret, la dynamique des proximités géographiques et organisées contribue à la création des territoires au sein desquels elles prennent naissance et se développent. La proximité géographique se rapporte à la distance entre les acteurs. Elle peut être recherchée ou subie. La proximité organisée concerne les différentes manières d'être proches qu'ont les acteurs (en dehors de la distance géographique). Elle repose sur deux logiques : la logique d'appartenance (appartenance au même graphe de relations) et la logique de similitude (adhésion mentale à des catégories communes) (Proximités territoriales : construire la gouvernance des territoires, entre conventions, conflits et concertations, Paris, Economica Anthropos, 2012). 
les prendre en compte. En effet, les acteurs interrogés plaident pour une nécessaire déclinaison de l'Économie turquoise. En cela, c'est une vision non homogène de mise en œuvre qui est revendiquée pour répondre à l'inexistence d'une homogénéité territoriale départementale. De façon paradoxale, cette représentation est partagée par la plupart des conseillers généraux et des agents du Conseil général ainsi que par des acteurs dont l'échelle d'action s'avère départementale, telle que CAD22. En effet, les élus que sont les conseillers généraux possèdent un mandat parfois cantonal, parfois communal et/ou intercommunal. Certains sont même référents GIZC. Ils sont donc rattachés à un territoire plus restreint que le département, territoire dont bien souvent ils défendent les intérêts avant même ceux du département. Pour autant, dans le cadre de l'Économie turquoise, leur échelle de référence reste l'échelle départementale, conformément au projet politique défini.

\subsection{Une représentation du territoire liée aux enjeux traités}

Un autre élément semble influencer la représentation qu'ont les acteurs : le département semble géographiquement trop étroit par rapport aux enjeux maritimes et littoraux. En effet, les axes de travail retenus au sein de la démarche ne sont pas propres à l'espace maritime et littoral costarmoricain. Nous reprendrons ici les termes d'un enquêté : "l'économie turquoise respecte des frontières administratives départementales, or, la mer n'a pas de frontières" (Chargé de mission, Association). Certains acteurs font ainsi remarquer que cette échelle représente un échelon qui n'est pas suffisamment englobant pour traiter des problématiques abordées. Pour eux, l'interrogation est d'autant plus forte qu'à l'échelle régionale de nombreux sujets sont traités : "Dans le maritime, comme dans d'autres domaines, il y a des échelles critiques. Le département est une échelle un peu juste maintenant par rapport à toutes ces activités où les choses se passent au moins au niveau régional» (Directeur, Établissement public). Les acteurs ayant cette représentation et l'exprimant travaillent à l'échelle supra-départementale ou bien, tout en possédant un 
ancrage local, agissent aussi à des échelons supra-départementaux comme c'est le cas pour le Comité Départemental des Pêches Maritimes et des Élevages Marins des Côtes-d'Armor (CDPMEM 22). Ainsi, la diversification de l'aquaculture, et plus particulièrement l'algoculture, constitue une priorité de la stratégie «Croissance Bleue» portée par l'Union Européenne et bénéficie de fonds importants dans le cadre de la Politique Commune de la Pêche 2014-2020. De nombreux projets sont menés sur l'ensemble du littoral breton et se trouvent coordonnés à l'échelle régionale au travers du programme Breizh'Alg. Piloté par le Conseil régional de Bretagne, ce programme stratégique a pour objectif le développement de la filière des algues en favorisant l'animation et en encourageant la mise en réseau des acteurs pour permettre l'émergence de projets. En ce sens, l'Économie turquoise doit se concevoir en lien avec ce type de politiques publiques et d'espaces de discussion déjà existants, abordant les problématiques littorales et maritimes.

\subsection{Une représentation du territoire liée à la compétence}

Un dernier élément influençant la représentation des acteurs est le champ d'intervention de l'Économie turquoise : le développement économique. La démarche ne relève pas d'une compétence attribuée au Conseil général, mais, au contraire, d'une politique volontariste de ce dernier. Or, dans le cadre des entretiens, la question de la compétence a régulièrement été abordée et semble influencer la représentation qu'ont les acteurs du territoire pertinent de mise en œuvre de la démarche : «Dans la mesure où le développement économique est une prérogative régionale, en quoi le Département décrète ou met en avant l'Économie turquoise?»(Directeur, entreprises). Ainsi, lorsque les acteurs interrogés décrivent l'Économie turquoise en s'attachant à chacune de ces orientations (Ports durables, Diversification de l'aquaculture, Forum de la Mer, etc.), il s'avère que, à leurs yeux, aucune ne relève directement du Département. «Si on prend le positionnement du Conseil général par rapport à chacune de ces thématiques, il n'y en a aucune qui soit réellement 
départementale et où le Conseil général puisse faire beaucoup de choses» (Directeur, Établissement public). Au contraire, les acteurs la présentent comme relevant de la prérogative régionale : «il [le Conseil général] a les ports alors même qu’il n’a pas les moyens du développement économique. Donc à un moment, soit ça remonte à la Région, soit on considère que les ports confiés au Département sont un outil social, sociétal, etc.» (Directeur, Entreprise).

Il nous faut donc revenir à l'Acte I de la décentralisation (1982-1983) qui donne à la Région l'essentiel de la compétence en matière d'intervention économique ${ }^{31}$ et les outils nécessaires à l'interventionnisme souhaité dans ce domaine, lui permettant de légitimer des pratiques d'interventions déjà anciennes, voire d'élaborer des politiques volontaristes d'aménagement ${ }^{32}$. Pour autant, alors même que les établissements publics de coopération intercommunale (EPCI) possèdent la compétence obligatoire de "développement économique», il n'est jamais fait mention de celles-ci dans le cadre des entretiens : les EPCI ne semblent pas être identifiés comme ayant cette compétence par les acteurs interrogés, ou du moins comme territoire pertinent de mise en œuvre d'une politique telle que celle de l'Économie turquoise. Au contraire, ce sont bien souvent les pays qui ont régulièrement été cités. Ces derniers sont identifiés par les acteurs comme interlocuteurs privilégiés de la Région dans le cadre de ses appels à projet « $\mathrm{GIZC}^{33}$ ».

Bien que les acteurs reconnaissent l'intérêt porté par le Conseil général à son espace maritime et littoral, la mise en œuvre de l'Économie turquoise se basant sur le territoire départemental

31 Patrick Le Galès, Gouvernement et gouvernance des territoires, Paris, La Documentation française, 2006.

32 Bernard Pecqueur, Le développement local : pour une économie des territoires, Paris, Syros, 2000.

33 Pour accompagner les structures publiques dans l'élaboration de lieux d'expression, de débats et de décisions, pour un développement durable de la zone côtière en Bretagne, un appel à projets GIZC a été lancé en 2011, en collaboration entre la Région Bretagne et l'État dans le cadre du contrat de projets État/Région 2007-2013, puis reconduit par la Région Bretagne en 2012 et 2013. 
n'est donc pas perçue comme pertinente. Cette représentation s'explique par le fait que sont confondus le Département, c'està-dire le Conseil général en tant que puissance publique avec des compétences attribuées, et le département en tant que territoire de gestion de cette même puissance publique. Cette représentation est exprimée tout à la fois par les acteurs, que ce soient des acteurs socio-économiques ou des collectivités territoriales (y compris, parfois, le Conseil général). Il nous faut néanmoins nuancer le propos. En effet, suite à une mission confiée à une conseillère générale, Marie-Reine Tillon, visant à établir un bilan de l'Économie turquoise, les conclusions de l'étude mettaient en avant la nécessité de poursuivre le portage politique par le Conseil général, dans un souci d'affichage départemental, mais aussi celle de confier le portage opérationnel à CAD22, acteur ayant la compétence développement économique, avec une nécessaire déclinaison prenant en compte les territoires d'action précédemment décrits.

Considéré comme prenant à la fois trop peu en compte les spécificités locales et étant, dans le même temps, trop restreint au regard des enjeux de l'espace maritime et littoral, le territoire convoqué pour mettre en œuvre l'Économie turquoise interroge. Les différentes représentations exprimées nous montrent que l'Économie turquoise relève de différents territoires, qu'ils soient d'actions ou politiques. Il devient alors très difficile de définir un unique territoire pertinent de mise en œuvre, les territoires d'action étant interdépendants ${ }^{34}$. Bien que l'Économie turquoise représente un projet de territoire politique pour le département des Côtes-d'Armor, elle relève de plusieurs échelles. Les territoires n'ont alors pas d'autre choix que de travailler ensemble : concevoir, élaborer et mettre en œuvre en coopération. Ainsi, comme le précise Martin Vanier «dans un monde complexe et ouvert, il n'existe pas d'échelle pertinente, et encore moins de territoires pertinents, seulement des politiques dont la pertinence est dans

$\overline{34}$ Martin Vanier, op. cit. 
la capacité à combiner les échelles et mobiliser tous les territoires ensemble ${ }^{35}$ ".

\section{L'interterritorialité de l'action comme réponse potentielle}

Ne pouvant se référer au seul département, la mise en œuvre de l'Économie turquoise impose de faire référence à l'ensemble des échelles, du global au local, territoires autant institués que d'actions, dans un souci d'articulation et de cohérence entre les différentes arènes de discussion et d'actions collectives. Ainsi, ce qui ressort à travers les propos des enquêtés, c'est le sentiment d'un manque d'articulation entre les espaces de discussion et d'actions collectives qui abordent les mêmes problématiques. Ce à quoi l'interterritorialité peut apporter une réponse.

\subsection{Le devoir d'articulation}

Il ne semble pas exister d'échelles ni de territoires pertinents pour la mise en œuvre de l'Économie turquoise. Plus encore, la Région à elle seule ne constitue pas une échelle pertinente. La prise en compte des spécificités locales passe alors par des appels à projet qu'elle initie dans le cadre des politiques qu'elle mène. Une politique telle que l'Économie turquoise doit se réfléchir en coordination car elle doit, tout à la fois, prendre en compte des spécificités locales tout en se retrouvant prise dans un ensemble plus vaste. Selon Neil Brenner, on peut parler de «devoir d'articulation", ou encore de "politique des échelles " ${ }^{36}$. C'est en cela que consiste l'interterritorialité. Ainsi, les politiques publiques territoriales ne doivent plus se concevoir et être conduites à l'échelle de territoires s'organisant en et pour eux-mêmes, mais au contraire, en interaction permanente avec les autres territoires aussi bien verticalement qu'horizontalement.

Linterterritorialité consiste donc en une dynamique de coopération entre territoires. Nous identifions ici deux types d'inter-

\footnotetext{
35 Martin Vanier, op. cit., p. 86

36 Neil Brenner, "Globalisation as Reterritorialisation : The Re-Scaling of Urban Governance in the European Union", Urban Studies, vol. 36, n 3, mars 1999, p. 431-451.
} 
territorialité qui semblent se dessiner. Tout d'abord une interterritorialité politique, qui relève plus de l'affichage et du discours politique. Ainsi, dès le rapport d'orientation de 2011 sur l'Économie turquoise, le Conseil général, en tant qu’institution politique, affirme l'articulation de sa démarche avec « la politique maritime européenne (livre bleu et livre vert), nationale (Grenelle de la Mer) et régionale avec la Charte des espaces côtiers bretons [...] et la conférence régionale de la mer et du littoral ${ }^{37}$ ». Cette articulation est toujours rappelée depuis lors dans les rapports d'étape sur la démarche, dans les articles de presse, etc. Pour autant, cette interterritorialité, bien que nécessaire comme préalable, ne signifie pas l'existence d'une articulation concrète dans la mise en œuvre. Le second type d'interterritorialité qui se dessine est ce que nous appelons une interterritorialité opérationnelle, bien qu'elle puisse aussi être qualifiée d'administrative. En effet, toute coopération commence déjà par l'articulation et la coopération entre administrations et équipes de projet. Pourtant, ce n'est pas une évidence et on peut observer des réflexes de pseudo-souveraineté, loin d'être le monopole des élus, quand bien même les administrations et services sont au service d'un projet politique. Ainsi, lors des premiers comités de pilotage de l'Économie turquoise, aucun représentant de la Région Bretagne n'était présent. Les membres du COPIL, parfois membres du Conseil général, faisaient valoir la nécessité d'inviter cette instance pour une meilleure articulation des actions menées, cette dernière étant trop peu visible. Elle a ainsi été récemment incluse au COPIL, mais l'articulation n'est pas aisée. Par exemple, lors de la production d'un document de travail établissant un bilan des actions menées dans le cadre de l'Économie turquoise et sur lequel les membres du COPIL étaient invités à participer, la Région a vivement réagi sur des actions qu'elle considérait être les siennes, comme relevant de sa compétence exclusive, alors que le document faisait seulement mention du Conseil général comme participant à ces actions, et non comme chef de file.

37 Conseil général des Côtes-d'Armor, Économie turquoise : Rapport d'orientations, op. cit., p. 8. 
Bien que l'interterritorialité puisse se présenter comme une réponse potentielle à une nécessaire articulation et coordination entre échelles, celles-ci se construisent sur le long terme. L'interterritorialité débute avec la prise de conscience qu'il n'y a pas d'autorité ou de chef de file unique, mais qu'au contraire, ces derniers peuvent changer en fonction des projets et des compétences. Un même projet peut aussi comporter différents chefs de file en fonction du phasage et de l'avancement du projet et des actions qui y seront menées. Néanmoins, on partage peu ce que l'on vient d'acquérir, encore moins lorsque les textes conservent une apparente répartition des compétences par niveaux de collectivités territoriales identifiées : "On parle de favoriser un développement global sur un territoire ayant une certaine légitimité, que ce soit un bassin de navigation ou un département; et puis on s'aperçoit que, dans les faits, chacun essaie de tirer la couverture à soi " (Chargé de mission, Association). Travailler en interterritorialité constitue bien souvent un changement culturel pour les services en charge de la mise en ouvre et demande l'abandon de pratiques habituelles. L'acculturation à cette pratique est une nécessité qui ne peut s'acquérir que sur le temps long, au travers de projets concrets permettant d'éprouver les difficultés et bienfaits d'une telle approche. Ainsi, bien qu'elle puisse être reconnue et revendiquée comme nécessaire, elle ne se concrétise pas toujours dans les faits, comme le reprochent certains acteurs interrogés dans le cadre de cette enquête sur l'Économie turquoise.

\subsection{Une interterritorialité encore peu visible}

La nécessité de réfléchir l'Économie turquoise dans une perspective interterritoriale est reconnue par le promoteur de la démarche qu'est le Conseil général. Cette volonté a fait l'objet d'un affichage, dès les tout premiers débuts de la démarche au travers du Rapport d'orientation de l'Économie turquoise produit en Automne 2011; ainsi que dans un article du magazine "Côtes d'Armor» de juillet-août 2011 où il était écrit : "Nous ne pouvons évidemment pas agir seuls et nous devons étendre cette vision à 
l'ensemble du territoire, de la région et au-delà ${ }^{38}$ ». L'emboîtement de la démarche dans un contexte global est donc conscientisé, tout comme la nécessité de prêter attention aux chevauchements et articulations existants. On peut alors supposer une maittrise de l'articulation des dispositifs. Cette dernière semble assumée par l'utilisation d'éléments formels (énonciation de l'articulation, documents officiels, etc.) et par la présence des mêmes acteurs aux différentes instances. Néanmoins, entre l'affichage et la réalisation, des divergences sont ressenties. "Je n'ai pas le sentiment que tout ce qui est Charte des Espaces Côtiers Bretons, Blue Growth, Assises de la mer, etc. s'imbriquent dans une vision commune. C'est toujours les mêmes personnes qui viennent, mais une fois terminé l'événement, la connexion n'est pas toujours évidente» (Directeur, Entreprise). Au-delà du discours, l'interterritorialité dans sa mise en ouvre opérationnelle via une coopération réelle des services reste peu visible; c'est-à-dire que plus que d'une question d'affichage, il s'agit d'un manque d'articulation entre les services et équipes de projet qui est ressenti. Pourtant, sans que la situation soit exemplaire, l'interterritorialité connaît des avancées progressives.

Deux initiatives sont intéressantes à présenter. Tout d'abord, celle de l'Assemblée des Départements de France qui a lancé, en 2013, la Mission Mer et Littoral avec pour ambition d'engager une réflexion «sur le renforcement du rôle des Départements sur ces territoires, en tant que garants des équilibres territoriaux ${ }^{39}$ " et "d'élaborer des propositions pour la mise en ouvre de politiques départementales de gestion intégrée des zones littorales et maritimes ${ }^{40}{ }$. Initiée par Claudy Le Breton, Président du Conseil général des Côtes-d'Armor, elle est présidée par Philippe Grosvalet, Président du Conseil général de Loire-Atlantique. Elle constitue un premier pas vers une interterritorialité horizontale en associant l'ensemble des Départements littoraux à élaborer des

\footnotetext{
38 Conseil général des Côtes-d'Armor, Économie turquoise. Terre et mer associées, Saint-Brieuc, Côtes d'Armor magasine, $n^{\circ}$ 100, juillet-août 2011, p. 12.

39 Assemblée des Départements de France, Invitation aux premiers Ateliers de la Mission Mer et Littoral, Philippe Grosvalet, 17 juin 2013, p. 1.

Ibid., p. 1.
} 
propositions de pistes d'actions opérationnelles pour engager l'amorce d'un changement dans la manière de "penser le littoral». La Mission Mer et Littoral répond en partie à certaines attentes exprimées par les acteurs interrogés. "Il faudrait que la réflexion Économie turquoise soit menée de manière synchronisée avec les autres départements bretons» (Directeur, Entreprise). Néanmoins, elle reste fixée sur «le périmètre d'intervention» supposé du Département : il s'agit de définir «une action départementale forte pour répondre aux enjeux maritimes et littoraux selon une approche intégrée et transversale, mais centrée sur ses périmètres d'intervention $^{41}$ ». De plus, bien que soit rappelée la nécessité de s'inscrire dans la gouvernance existante, de ne pas vouloir tout gérer, le département est présenté comme l'«échelon territorial pertinent et adapté à une logique territoriale prenant en compte les relations entre le littoral, le rétro-littoral et l'arrière-pays ${ }^{42}$ ", alors que la réelle pertinence consisterait en un politique organisée conjointement à plusieurs échelles, selon des moyens, des rôles et des pouvoirs complémentaires ${ }^{43}$. La deuxième initiative intéressante en termes de coopération interterritoriale est la Conférence Régionale de la Mer et du Littoral initiée par la Région Bretagne dans le cadre de sa Charte des Espaces Côtiers Bretons. Cette instance a notamment l'ambition d'être un lieu d'échanges, de réflexions et d'actions de l'ensemble des acteurs bretons de la zone côtière. Elle réunit les collectivités locales et leurs groupements, l'État et les établissements publics liés à la mer, les organisations socioprofessionnelles et le milieu associatif. Aujourd'hui devenue le lieu de débat privilégié et de concertation pour les questions liées à la mer et au littoral en Bretagne, elle est reconnue comme une instance officielle par les différentes collectivités sur tous les dossiers en lien avec le littoral et l'espace maritime. Elle représente tout à la fois un lieu d'interterritorialité opérationnelle et politique et constitue une instance centrale pour tout dossier ayant trait à l'espace maritime et littoral breton.

41 Conseil général de Loire-Atlantique, Lettre d'information $n^{\circ} 2$. Mission Mer et

Littoral, Nantes, décembre 2013.

43 Martin Vanier, op. cit. 
Enfin, au-delà de ces initiatives très larges et visibles, il en existe de plus discrètes. Ainsi, dans le cadre de l'Économie turquoise, sont intégrés au COPIL la Région Bretagne et les chargés de mission (GIZC), en réponse à cette volonté de renforcer l'articulation entre territoires dans un souci d'interterritorialité.

\section{Conclusion}

L'Économie turquoise, projet de territoire initié à l'échelle départementale par le Conseil général des Côtes-d'Armor procède d'une représentation du territoire départemental, auquel a été adjoint l'espace maritime et littoral, comme échelle pertinente de mise en ouvre. Pour autant, le partage d'une telle conception ne fait pas l'unanimité. En effet, cette politique recouvre des territoires multiples tant du point de vue des représentations, que de l'identité ou de l'appropriation de l'espace, notamment en termes d'usages. C'est aussi une politique qui s'intègre dans un contexte plus global, tant dans l'évolution de la perception des espaces maritimes et littoraux que des politiques menées à leur égard. Enfin, en tant que politique de développement économique, elle va bien au-delà des seules compétences attribuées aux Conseils généraux (compétence relevant des Conseils régionaux et des EPCI) et représente ainsi une politique volontariste. Néanmoins, bien que le Conseil général souhaite s'appuyer sur le territoire départemental pour apporter une réponse, à son échelle, aux enjeux traités au sein de l'Économie turquoise, bien qu'il soit convaincu d'être souverain à son échelle; est exprimée la conscience d'une nécessaire articulation entre les scènes de discussion et d'action collective présentes à la fois à l'échelle des territoires de pratiques et d'action locaux, mais aussi supradépartementaux. En cela, il rejoint les différentes préoccupations exprimées par les acteurs ne partageant pas son point de vue dans sa globalité. En effet, l'essence de l'action collective territoriale est interterritoriale. Ceci est d'autant plus vrai en ce qui concerne l'espace maritime où les limites spatiales définies interfèrent avec les limites des compétences autant verticalement qu'horizontalement. Bien que la gestion de cet espace relève de l'État, ce dernier 
n'est pas, pour autant, déconnecté du terrestre dont la gestion relève de divers échelons de collectivités territoriales (la gestion de la qualité de l'eau en est un parfait exemple). La conception des politiques publiques, tout particulièrement sur les espaces maritimes et littoraux, ne peut donc pas relever d'un seul échelon, d'un seul territoire. Bien au contraire, les enjeux y prenant place ne sont pas propres à un territoire en particulier et leur gestion ne relève pas de compétences attribuées à un seul échelon de collectivité. Les usages y prenant place sont multiples et chaque territoire, à son échelle, y mène actions et politiques avec un risque accru de redondance, voire d'empiètement. La conception et la mise en œuvre de politiques sur ces espaces doivent donc être conduites en interaction permanente entre territoires, autant verticalement qu'horizontalement. L'Économie turquoise, en débordant du département et des compétences attribuées au Conseil général, doit se réfléchir dans une perspective interterritoriale de l'action pour répondre aux enjeux de prise en considération des différents territoires d'usages et de pratiques mobilisés par le projet et aux enjeux de nécessaire coopération entre les différentes collectivités concernées. Pour autant, l'interterritorialité ne représente pas toujours une évidence et procède d'une construction s'installant dans la durée. En effet, elle demande l'abandon d'habitudes plus ou moins ancrées individuellement et collectivement, de jeux de pouvoirs habituels. Dans ces conditions, on peut se demander comment dépasser ces difficultés et quelles sont les conditions favorisant l'interterritorialité. 


\section{Bibliographie}

Angeon, Valérie et Nathalie Bertrand, Les dispositifs contractuels de développement rural: quelles proximités mobilisées?, Colloque ASRDLF sur Les dynamiques territoriales : débats et enjeux entre les différentes approches disciplinaires, Grenoble-Chambery, 10-12 juillet, 2007.

Assemblée des Départements de France, Invitation aux premiers Ateliers de la Mission Mer et Littoral, Philippe Grosvalet, 17 juin 2013.

Assemblée des Départements de France, Synthèse des échanges - Mission mer et littoral-Atelier 2, 4 et 5 novembre 2013.

Balandier, Georges, Le désordre : éloge du mouvement, Paris, Fayard, 1988.

Balandier, Georges, Anthropologie politique, Paris, Presses universitaires de France, coll. "Quadrige», 1995.

Bécet, Jean-Marie, L'aménagement du littoral, Paris, Presses universitaires de France, coll. «Que sais-je?», 1987.

Boutinet, Jean-Pierre, Anthropologie du projet, Paris, Presses universitaires de France, coll. "Quadrige», 2012.

Brenner, Neil, "Globalisation as Reterritorialisation: The Re-Scaling of Urban Governance in the Uuropean Union", Urban Studies, vol. 36, $\mathrm{n}^{\circ} 3$, mars 1999, p. 431-451.

Comité Interministériel de la Mer, Livre bleu - Stratégie nationale pour la mer et les océans, Paris, 2009.

Commission européenne, Blue Growth : opportunities for marine and maritime sustainable growth, Bruxelles, Direction générale des affaires maritimes et de la pêche, 2012.

Conseil de l'Europe, Modèle de loi sur la gestion durable des zones côtières et Code de conduite européen des zones côtières, 1999.

Conseil général de Loire-Atlantique, Lettre d’information $n^{\circ}$ 2. Mission Mer et Littoral, Nantes, décembre 2013.

Conseil général des Côtes-d'Armor, Compte-rendu du groupe de travail élargi «Économie turquoise», Saint-Brieuc, 18 octobre 2011.

Conseil général des Côtes-d'Armor, Compte-rendu du groupe de travail élargi «Économie turquoise», Saint-Brieuc, 12 janvier 2012.

Conseil général des Côtes-d'Armor, Compte-rendu du groupe de travail élargi «Économie turquoise», Saint-Brieuc, 10 juillet 2012.

Conseil général des Côtes-d'Armor, Économie turquoise. Rapport d'orientations, Saint-Brieuc, Direction de la mer et du littoral, $\mathrm{n}^{\circ} 4.5$, Séances des 22 et 23 septembre 2011.

Conseil général des Côtes-d'Armor, Initiative thématique "Mer et Littoral", Saint-Brieuc, Côtes d'Armor 2mille20, 2006. 
Conseil général des Côtes-d'Armor, Économie turquoise. Terre et mer associées, Saint-Brieuc, Côtes d'Armor magasine, no ${ }^{\circ}$ 100, juillet-août 2011, p. 11-13.

Conseil général des Côtes-d'Armor, Contribution à une stratégie départementale de Recherche et d'Innovation en Côtes-d'Armor, Saint-Brieuc, 2011

Conseil général des Côtes-d'Armor, Faire de la mer et du littoral une force pour notre département, Budget primitif 2012, Saint-Brieuc, Séances des 27, 28 février, 1 et 2 mars 2012.

Délégation à l'aménagement du territoire et à l'action, Le Littoral français : perspectives pour l'aménagement, Paris, la Documentation française, 1972.

Gerbaux, Françoise (dir.), Utopie pour le territoire : cohérence ou complexité, La Tour-d'Aigues, Éditions de l'Aube, 1999.

Gob, Rosaire, «Le management du territoire par le projet : des limites de l'instrumentation gestionnaire. Le cas de la Guadeloupe», Thèse, Conservatoire National des Arts et Métiers, 2012.

Gumuchian, Hervé et al., Les acteurs, ces oubliés du territoire, Paris, Economica Anthropos, 2003.

Lajarge, Romain, "Pas de territorialisation sans action (et vice versa)?", dans Martin Vanier (dir.), Territoires, territorialité, territorialisation: controverses et perspectives actes des entretiens de la Cité des territoires, Grenoble, 7 et 8 juin 2007, «Territoires, territorialité, territorialisation... et après?", Rennes, Presses universitaires de Rennes, 2009, p. 193-204.

Le Galès, Patrick, Gouvernement et gouvernance des territoires, Paris, La Documentation française, 2006.

Lozachmeur, Olivier, «Le concept de gestion intégrée des zones côtières : le point de vue d'un juriste», Océanis, vol. 30, n 1, 2004, p. 5170.

Merckelbagh, Alain, Et si le littoral allait jusquâ la mer !, Versailles, Éditions Quae, 2009.

Moine, Alexandre, "Comprendre et observer les territoires : l'indispensable apport de la systémique», Habilitation à Diriger des Recherches, Université de France-Comté, 2014.

Pecqueur, Bernard, Le développement local : pour une économie des territoires, Paris, Syros, 2000.

Péron, Françoise et Jean Rieucau, La maritimité aujourd'hui, Paris, L'Harmattan, 1996.

Roux, Michel, Limaginaire marin des Français : mythe et géographie de la mer, Paris, L'Harmattan, 2000. 
Sonnic, Ewan, «La navigation de plaisance : territoire de pratique et territoires de gestion en Bretagne, entre dualité et nécessité de fusion pour une évolution progressiste de l'activité», Thèse, Université de Rennes 2, 2005.

Torre, André et Jean-Eudes Beuret, Proximités territoriales : construire la gouvernance des territoires, entre conventions, conflits et concertations, Paris, Economica Anthropos, 2012.

Vanier, Martin, Le pouvoir des territoires : essai sur l'interterritorialité, préface de Philippe Estèbe, Paris, Economica Anthropos, 2008.

Vanier, Martin (dir.), Territoires, territorialité, territorialisation : controverses et perspectives, Rennes, Presses universitaires de Rennes, coll. Espace et territoires, 2009. 